\title{
Computer Aided Planning in Construction Survey and Analysis of Construction Planning Procedures
}

Naruo Kano

Department of Architecture, Waseda University

3-4-1 Okubo, Shinjuku, Tokyo, 169 JAPAN

\begin{abstract}
In this paper, the author conducted surveys on two construction planning processes in an attempt to establish a system of CAP (Computer Aided Planning) for construction projects. The surveys were followed by a discussion of the structure and characteristics of planning. The author concluded that current systems for computer-aided construction planning do not provide all functions that engineers need to develop construction plans, and indicated twelve functions required for a computer aided construction planning system.
\end{abstract}

\section{Introduction}

Since the PERT/CPM technique was developed in the late 1950's, many computer programs for construction planning have been developed and implemented. With these systems, construction engineers are now able to perform efficient, detailed analysis of temporary structural analysis, network calculation, process simulation, quantitative surveys, and documentation associated with construction planning tasks. Despite such dedicated computer systems, however, practical construction planning still depends largely on the individual knowledge and experience of engineers involved in the planning.

A.Laufer(1990)1), and A.Shapira(1994)2), O.O.Faniran(1994)3) have discussed the importance of construction planning and pointed out, effects of uncertainties, planning activities in various stages, and the correlation between the efforts and their effectiveness in planning. However, the philosophies and methodologies needed to provide effective assistance for construction planning have yet to be identified.

In this paper, the author conducted surveys on two construction planning processes in an attempt to establish a system of CAP (Computer Aided Planning) for construction projects. The surveys identified the nature and sequence of empirical planning methods used by engineers and showed that these procedures depend largely on expertise and experience. The author discussed the characteristics of the planning process and defined the conditions for use of CAP in construction projects and the functions required of such a system. 


\section{Planning for Building Construction}

\subsection{Plan Contents}

The building construction process consists of various jobs that proceed chronologically with complex interactions. At the site, different types of workers and equipment must be allocated on time, in order, and in the proper amounts for each day of the construction period. Therefore, construction planning must deal with such issues as equipment selection, handling of materials, and equipment layout at the site as well as the sequence and method of operations.

\subsection{Plan Structure}

In construction planning, engineers must select optimum methods from the available alternatives, basing their decisions on drawings, specifications, and various constraints that might be expected from scheduling and site layout. Moreover, engineers must find the optimum combination of methods, workers, materials, equipment, and scaffolding. Uncertainties such as weather and soil conditions at the site must be taken into account when making these decisions.

To design a construction plan that is consistent as a whole and takes into account any uncertainties, engineers must be able to grasp both the whole plan and its details. To achieve this, the construction plan should be divided into three levels: Master Plan, Working Plan, and Detailed Work Plan. These should be developed simultaneously during the planning phase.

\subsection{Plan Complexity}

The delay in the computerization of planning can be traced to two factors. Since such a variety of plan items must be decided, the planning system must encompass a broad range of expertise as well as specialized technical knowledge to solve the problem. To optimize the plan, the system must determine the most appropriate combinations from an infinite number of feasible solutions. Such a task is nearly impossible even with computers; instead, it requires the use of heuristic algorithms.

Also contributing to the delay are the complex interrelationships between elements in the planning process. In order to consider one element in the process, the system must also take into account a number of related elements. This means that most parts of a plan depend on other parts and must be resolved simultaneously, as when solving simultaneous equations. Obviously, computer systems cannot deal with huge problems based on complex logic. Thus, engineers are forced to use a trial-and-error approach with certain heuristic procedures.

\section{Surveys on Construction Planning}

\subsection{Overview}

The author conducted two surveys to investigate construction planning for building projects. The first, "Case I," focused on the experimental planning procedure for an already-built eight-story office building with one basement and a total floor area of $4,000 \mathrm{~m}^{2}$. An experienced construction engineer was responsible for designing a mock master plan for the project, but was given no information about the actual construction project. 


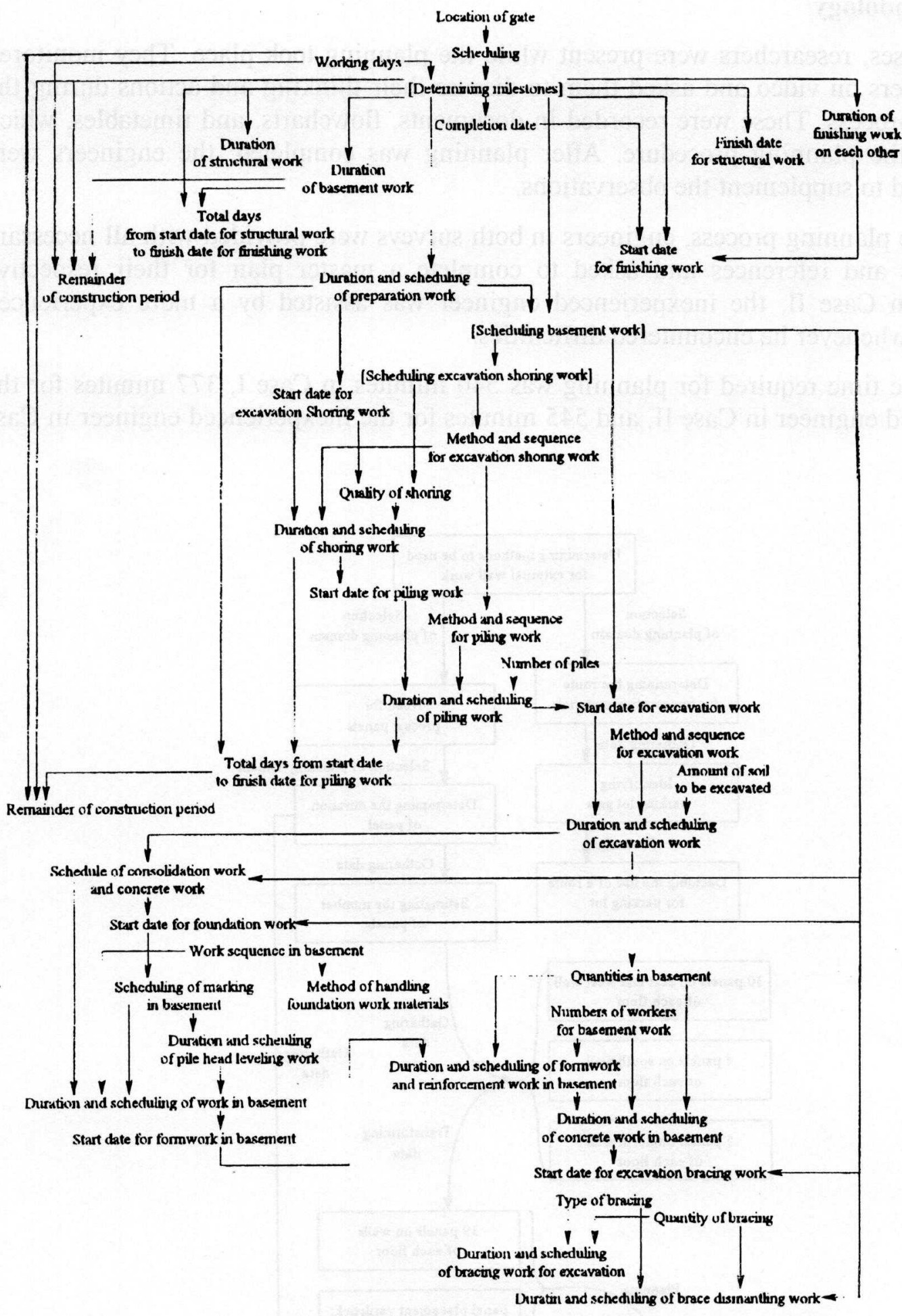

Figure 1 Planning Procedure for Case I

The second survey, "Case II," focused on the pre-tender planning for an ongoing project for a eight-story hotel building with one basement and a total floor area of $1,000 \mathrm{~m}^{2}$. Two engineers -- one experienced and one inexperienced -- were selected to identify differences in their planning procedures. The first survey was conducted in 19914); the second, in 1994. 


\subsection{Methodology}

In both cases, researchers were present while the planning took place. They monitored the engineers on video and asked them to discuss their thinking and actions during the planning process. These were recorded in documents, flowcharts, and timetables, which represent the planning procedure. After planning was completed, the engineers were interviewed to supplement the observations.

During the planning process, engineers in both surveys were provided with all necessary documents and references and asked to complete a master plan for their respective projects. In Case II, the inexperienced engineer was assisted by a more experienced colleague whenever he encountered difficulties.

Cumulative time required for planning was 540 minutes in Case I, 377 minutes for the experienced engineer in Case II, and 545 minutes for the inexperienced engineer in Case II.

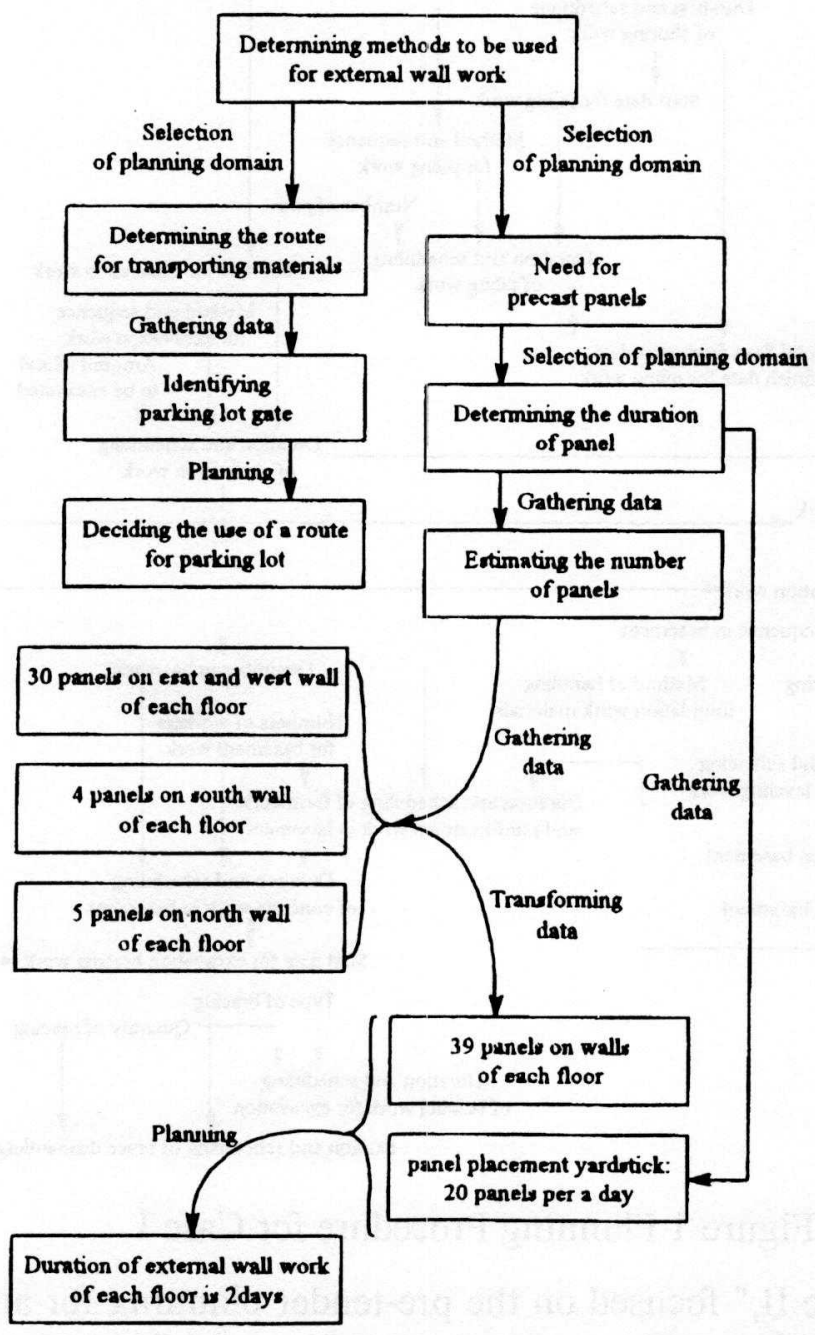

Figure 2 Planning Sequence for External Wall Work 


\subsection{Results}

\subsubsection{Case I}

In this survey, the author investigated the planning process and sequence. The engineer began by creating a framework of plan, including milestones and the duration of major jobs, before working out the details. After the framework had been established, it functioned as a goal for the planning process. The engineer repeatedly modified this framework to reflect the current state of the detailed plan.

Figure 1 shows the planning process. The engineer started by deciding the location for site access gates and determining the major milestones and duration for structural work on each floor. During planning, the duration of structural and finishing work was deduced from the total duration for the project. After a framework had been completed, planning efforts were directed to the details of foundation and excavation work, which would represent a large percentage of the total temporary construction expenses and where the engineer has a great deal of freedom.

Figure 2 depicts the planning process for external walls, which consist of precast panels. This process started at the point where the engineer decided to consider this plan. The gate and transportation route for the panels were first considered, and access to the materials was then confirmed. Attempting to determine the duration of external wall work, the engineer recognized the need for productivity data for panel erection using a crane. Based on the drawings, he compiled data on the total number of panels and estimated a yardstick value based on personal experience. In this way the duration of the external wall work was determined.

\subsubsection{Case II}

This survey aimed to identify differences in the planning process resulting from professional experience. Significant differences were observed between the two engineers in terms of planning sequence and methodology. These are summarized below.

\section{a. Experienced Engineer}

Time for planning tasks estimated and allocated in advance

More time spent studying drawings and specifications

Recognition for critical parts of the plan

Less time spent documenting the output

Top-down approach

b. Inexperienced Engineer

Repeated modifications to plans

Excessive focus on detail

Insufficient scope of constraints to be considered

Excessive modifications during the documentation stage

Bottom-up approach 
While the inexperienced engineer developed the plan based primarily on the sequence of construction, the experienced engineer started with major elements of the plan, such as structural work, proceeded to finishing work, and then returned to preparation and excavation work. This was because he knew from study of the initial drawings and specifications that structural and finishing work would be critical to this project. Such inference and intuitive judgment is an important vehicle for the development of effective, appropriate plans.

\section{Analysis of Construction Planning}

\subsection{A Conceptual Model of Planning}

\subsubsection{Planning Information}

The surveys showed that the items of information used in construction planning can be divided into four categories:

(1) Goals: Items indicating a direction and strategy for planning

(e.g., mandatory period, milestones)

(2) Constraints: Conditions that the plan must satisfy

(e.g., drawings, specifications, site condition)

(3) Plans: Elements of the whole plan

(e.g., construction method, schedule, site layout)

(4) Status: Items subject to other items and representing the status of the plan

(e.g., total number of workers, productivity)

In construction planning, goals indicate expected results, and the variance between expected status and current status offers a valuable criterion for evaluating the planning. Engineers design plans to achieve certain goals in light of certain constraints, producing plan elements that take into account the whole plan together with status items indicated later by other items.

\subsubsection{Relationships Among Items}

When a construction plan is broken down into smaller elements, it can be represented as a set of discrete but interrelated information items. In planning the formwork method, for example, drawings and specification affecting the method must be examined. If the flying-shore method is selected for the formwork, this will affect the selection of a crane to be used at the site, since the crane must have sufficient capacity to lift the shores effectively.

Figure 3 shows an example of relationships in a plan, where drawings and specifications represent constraints and mandatory construction period, a goal, both of which have interrelationships that affect the formwork method. The method also affects the duration of formwork and the number of workers required. The duration, in turn, affects the total construction period, which must satisfy the goal mentioned above. This chain of effects forms a loop. 


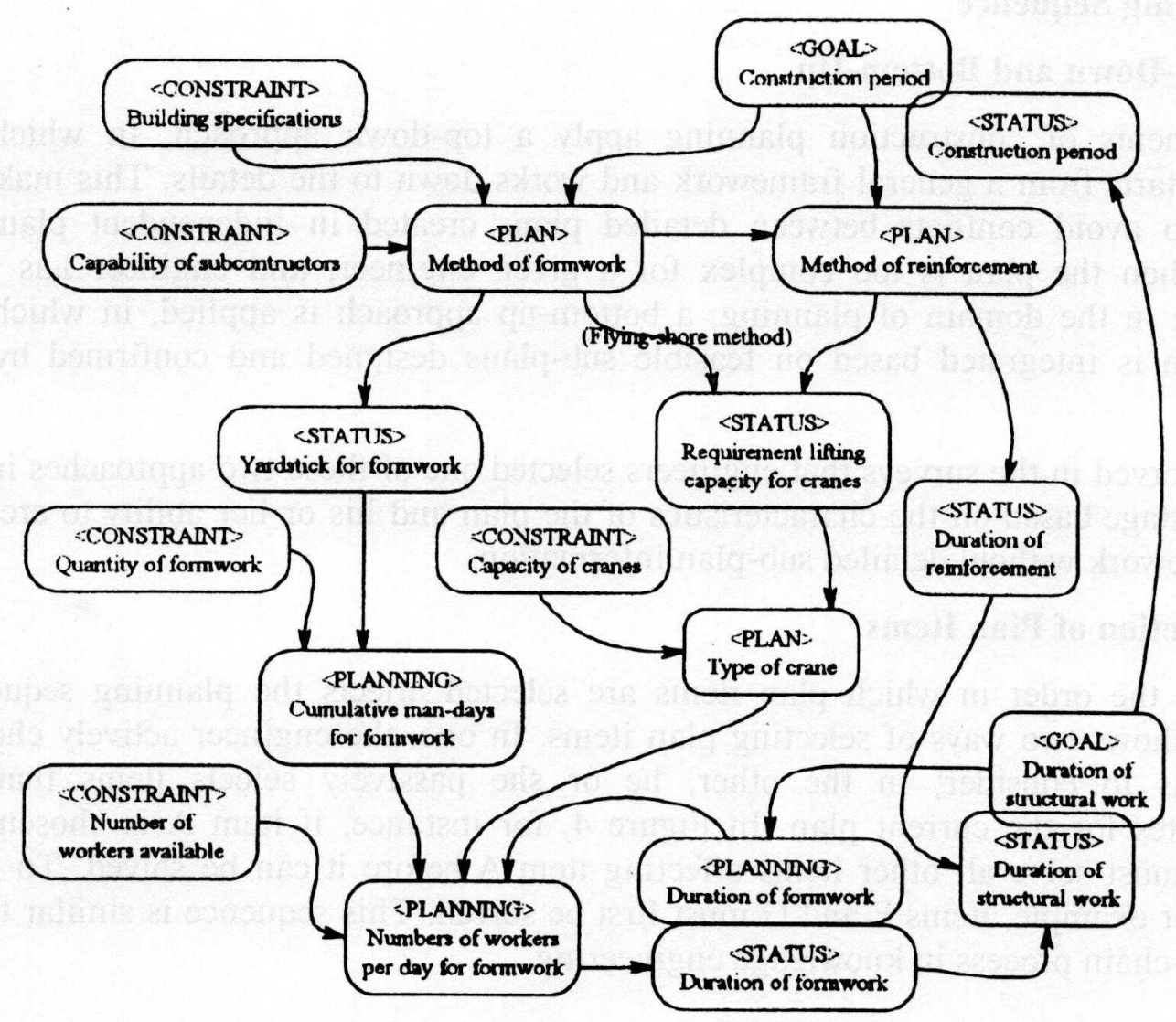

Figure 3 Relationships Among Information Items in Formwork Planning

\subsection{Planning Functions}

The survey results showed that construction planning consists of the six functional tasks shown below. Each plays a role based on information provided by other functional tasks in the plan.

(1) Establishment of planning goals

(2) Collection of data

(3) Compilation and analysis of data

(4) Selection of planning domain

(5) Planning

(6) Evaluation of plans

\subsection{Imagining the Construction Process}

Planning follows the construction process. Thus, planning depends largely on an engineer's image of the construction process and ability to retain and manipulate associated data. The surveys showed that engineers drew up plans using a mental picture of the site where the work was to be done. This is why engineers prefer to design plans that follow the actual construction process. 


\subsection{Planning Sequence}

\subsubsection{Top-Down and Bottom-Up}

Most elements of construction planning apply a top-down approach, in which an engineer starts from a general framework and works down to the details. This makes it possible to avoid conflicts between detailed plans created in independent planning stages. When the plan is too complex for a given engineer, and engineer has little experience in the domain of planning, a bottom-up approach is applied, in which the entire plan is integrated based on feasible sub-plans designed and confirmed by the engineer.

It was observed in the surveys that engineers selected one of these two approaches in the planning stage based on the characteristics of the plan and his or her ability to create a plan framework without detailed sub-plan information.

\subsubsection{Selection of Plan Items}

Naturally, the order in which plan items are selected affects the planning sequence. Figure 2 shows two ways of selecting plan items. In one, the engineer actively chooses plan items to consider; in the other, he or she passively selects items that are prerequisites for the current plan. In Figure 4 , for instance, if item $\mathrm{A}$ is chosen, the engineer must solve all other items affecting item A before it can be solved. To solve item $\mathrm{C}$, for example, items $\mathrm{F}$ and $\mathrm{G}$ must first be solved. This sequence is similar to the backward-chain process in knowledge engineering.

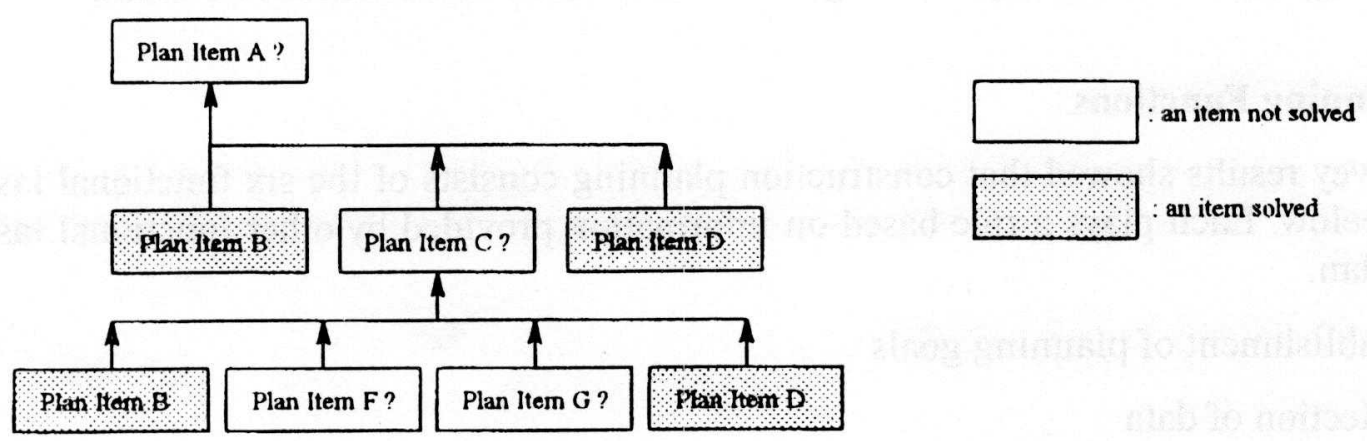

Figure 4 Selection of Plan Items

The second method is adopted when a planner finds a plan item for which all prerequisite items have been solved. In Figure 4 , if plan items $F$ and $G$ have been determined in the planning stage, an engineer might recognize that items $\mathrm{C}$ and $\mathrm{A}$ can be solved, in this order, based on the information at hand. Even if the engineer is planning the relevant parts for items $F$ and $G$, it might be better to solve the another parts: items $\mathrm{C}$ and $\mathrm{A}$ at this point, because the engineer would keep the planning values in mind for only a limited time after these planning activities. This is similar to the forward-chain process in knowledge engineering.

In most cases, experienced engineers use the former method, but the second method is used for some plan elements to improve efficiency. 


\subsubsection{Planning Priorities}

In the survey, engineers in construction planning were given an expected completion time and were not allowed sufficient time, which is similar to actual situations. The engineers were concerned about delays in their work because of unexpected difficulties in planning. The surveys showed that, under these circumstances, engineers began by planning essential elements that have a larger impact on planning results in terms of cost and time. They were apt to assign higher priority to plan items with a substantial effect on the criteria, executing them as soon as possible and leaving lower-priority items to be solved later.

\section{Essentials for Computer Aided Planning in Construction}

The surveys showed that engineers proceeded by grasping both the scope and details of the plans and then clarifying the ambiguities and integrating them into a solid whole. In the progress, engineers used images of the site as expected according to their plans, identifying problems and resolving them. This suggests that engineers do not have a fixed planning procedure but instead proceed in a sequence that they see as being most appropriate for the goal and current plans.

It is obvious that conventional computer-aided planning systems are not fully capable of helping an engineer proceed efficiently with planning and produce a proper plan. Instead, they are designed to provide support only in the form of databases, analysis, and documentation.

The survey results show that computer-aided systems for construction planning should have twelve functions to assist planning tasks:

(1) Supply information and knowledge when required

(2) Store ideas and key points of the planner and display them when necessary

(3) Calculate and analyze complex problems

(4) Model plan alternatives

(5) Infer detailed plans from master plans

(6) Infer master plans from detailed plans

(7) Retrieve past construction records

(8) Provide engineers with relevant information

(9) Simulate situations based on current plans

(10) Support the planner's imagination for the site situations

(11) Produce documents presenting plan alternatives

(12) Evaluate plan alternatives

\section{Conclusion}

In this paper, the author conducted two surveys on construction planning practices and discussed the structure and characteristics of planning. These surveys concluded that 
current systems for computer-aided planning have yet to provide the functions that engineers need to develop construction plans. Finally, the author identified twelve functions required for a computer-aided planning system in construction.

\section{References}

[1] A.Laufer, et al: Factors Affecting Construction Planning Outcomes, J.of Construction Engineering and Management, Vol.116, No.1, 1990, pp135-156

[2] A.Shapira, et al: Evolution of Involvement and Effort in Construction Planning throughout Project Life, Int. J. of Project Management, Vol.11, No.3, 1993.08, pp 155-164

[3] O.O.Faniran, et al: Effective construction Planning, Construction Management and Economics, 1994, 12, pp485-499

[4] N.Kano, et al: A Study on the Process of Construction Planning, Proc. of 'Kenchikuseisan to kanrigijutu' Panel Discussion, 1994.02, pp1-8 (in Japanese) 